\title{
Size-dependent symmetry breaking in models for morphogenesis
}

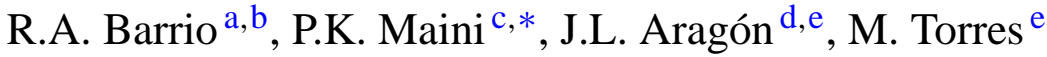 \\ a Instituto de Física, Universidad Nacional Autónoma de México, Apartado Postal 20-364, Mexico 01000, Distrito Federal, Mexico \\ ${ }^{\mathrm{b}}$ Department of Theoretical Physics, Oxford University, 1 Keble Road, Oxford OX1 3NP, UK \\ ${ }^{c}$ Centre for Mathematical Biology, Mathematical Institute, Oxford University, 24-29 St. Giles, Oxford OX1 3LB, UK \\ ${ }^{\mathrm{d}}$ Centro de Física Aplicada y Tecnología Avanzada, Universidad Nacional Autónoma de México, \\ Apartado Postal 1-1010, Querétaro 76000, Mexico \\ e Instituto de Física Aplicada, Consejo Superior de Investigaciones Científicas, Serrano 144, 28006 Madrid, Spain
}

\begin{abstract}
A general property of dynamical systems is the appearance of spatial and temporal patterns due to a change of stability of a homogeneous steady state. Such spontaneous symmetry breaking is observed very frequently in all kinds of real systems, including the development of shape in living organisms. Many nonlinear dynamical systems present a wide variety of patterns with different shapes and symmetries. This fact restricts the applicability of these models to morphogenesis, since one often finds a surprisingly small variation in the shapes of living organisms. For instance, all individuals in the Phylum Echinodermata share a persistent radial fivefold symmetry. In this paper, we investigate in detail the symmetry-breaking properties of a Turing reaction-diffusion system confined in a small disk in two dimensions. It is shown that the symmetry of the resulting pattern depends only on the size of the disk, regardless of the boundary conditions and of the differences in the parameters that differentiate the interior of the domain from the outer space. This study suggests that additional regulatory mechanisms to control the size of the system are of crucial importance in morphogenesis.
\end{abstract}

() 2002 Elsevier Science B.V. All rights reserved.

PACS: 82.40.Ck; 47.54.+r; 05.45.-a

Keywords: Pattern formation; Reaction-diffusion system; Morphogenesis; Mathematical biology

\section{Introduction}

On 12 March 1832, Michael Faraday wrote an observation deposited at the Royal Society of London: "I am inclined to compare the diffusion of magnetic forces from a magnetic pole to the vibrations upon the surface of disturbed water, or those of air in the phenomenon of sound, i.e., I am inclined to think that the vibratory theory will apply to these phenomena as it does to sound, and most probably light".

\footnotetext{
* Corresponding author. Tel.: +44-1865-280617; fax: +44-1865-270515.

E-mail address: maini@maths.ox.ac.uk (P.K. Maini).
}

This remarkable sentence was undoubtedly dictated to this great mind by the amazing similarity of wave patterns formed in wildly different systems (either acoustical, optical, hydrodynamic, or chemical), and envisaged the universality of the classical theory of oscillations, to be fully developed many years later. A completely analogous situation occurs in the birth, development, and stabilization of patterns described by nonlinear dynamical systems [1], where the appearance of regular symmetric patterns very often present similar shapes, independently not only of the physical system, but also of the mechanisms that drive the spontaneous symmetry breaking. 
A beautiful, although complicated, example of this is the Faraday experiment [2], where a spatio-temporal regular pattern is parametrically produced through a combined Turing-Hopf bifurcation [3]. This experiment is deceptively simple and consists of oscillating vertically a vessel containing a fluid with a certain amplitude and frequency. We have performed the Faraday experiment using a cylindrical vase containing a very special fluid with high molecular weight [4,5], and were able to obtain extremely robust regular patterns, of practically any azimuthal symmetry, by varying the bifurcation parameter. In that paper we derived a theoretical model which clearly shows a parametric term and a Turing-like structure. Furthermore, by following the bifurcations of the fivefold patterns we pointed out that their structures resemble the shell shape of sea urchins in different geological times [4].

This observation immediately poses two important questions: (1) What is the link between waves on the surface of a liquid and the shape of living organisms? (2) How is it possible to robustly select a given symmetry from a wide choice of patterns? The answer to the first question is undoubtedly due to the fact that the universality of the symmetry-breaking properties encompasses a large range of different dynamical systems. This paper shall be mostly concerned with showing this. The second question is more difficult to answer since, as we shall discuss later, it should involve the interaction between the pattern-forming system and another mechanism, capable of controlling the path of bifurcations.

Since we are mentioning the formation of symmetrical patterns in living organisms, let us concentrate our effort on a simple Turing system. Turing [6] proposed his famous equations to "discuss a possible mechanism by which the genes of a zygote may determine the anatomical structure of the resulting organism", coining the term "morphogenesis". This idea has been extensively used in many developmental and ecological problems [7] (for a review, see Ref. [8]). More about Turing systems can be found in Ref. [9].

Bifurcation analysis and numerical calculations of Turing models in one and two dimensions show that, in the main, they produce very similar patterns, regardless of the specific form of the reaction terms and of the significance of the nonlinear parameters. Therefore, we propose that, in a circular domain, the quantity that defines the symmetry of the pattern is the radius of the circle. The specific model we choose to study in detail has been published elsewhere [10]. There, it was shown that it produces the typical stripes or spots of general Turing models, depending on the values of the nonlinear parameters. It was verified that a cubic term favors stripes, while the presence of a quadratic term produces spots, and that spots are much more robust than stripes.

In the following section we present the model and a linear analysis when the shape of the domain is circular. We also analyze the case of matching boundary conditions, when the circle is embedded in a square domain. Then, we show several numerical results that corroborate our hypothesis, and we draw some conclusions in the last section.

\section{The model}

Turing equations describe the temporal development of the concentrations of two chemicals, $U$ and $V$, that diffuse at different rates $\left(D_{U}=D\right.$ and $\left.D_{V}=1\right)$, and react according to the nonlinear functions $f$ and $g$ :

$$
\begin{aligned}
& \frac{\partial U}{\partial t}=D \nabla^{2} U+f(U, V), \\
& \frac{\partial V}{\partial t}=\nabla^{2} V+g(U, V) .
\end{aligned}
$$

The model equations are obtained by expanding the nonlinear functions in a Taylor series around the stationary uniform solution $\left(U_{\mathrm{c}}, V_{\mathrm{c}}\right)$, up to cubic terms [10]:

$$
\begin{aligned}
& \frac{\partial u}{\partial t}=D \delta \nabla^{2} u+\alpha u\left(1-r_{1} v^{2}\right)+v\left(1-r_{2} u\right), \\
& \frac{\partial v}{\partial t}=\delta \nabla^{2} v+\beta v\left(1+\frac{\alpha r_{1}}{\beta} u v\right)+u\left(\gamma+r_{2} v\right),
\end{aligned}
$$

where $u=U-U_{\mathrm{c}}$ and $v=V-V_{\mathrm{c}}$. The quantity $\delta$ conveniently gives the size of the system and the particular arrangement of the coefficients obeys conservation rules between chemicals. In order to keep the solutions as simple as possible, we enforce $(0,0)$ to 
be the only spatially uniform steady state by setting $\alpha=-\gamma$.

We shall investigate the conditions to set up a diffusion-driven instability in a two-dimensional disk with zero-flux boundary conditions. Near the steady state, $u=\mathrm{d} u$ and $v=\mathrm{d} v$ and the linearized system reads

$\frac{\partial \mathrm{d} u}{\partial t}=D \delta \nabla^{2} \mathrm{~d} u+\alpha \mathrm{d} u+\mathrm{d} v$,

$\frac{\partial \mathrm{d} v}{\partial t}=\delta \nabla^{2} \delta v+\beta \mathrm{d} v-\alpha \mathrm{d} u$.

In a circular domain, we look for solutions of the form

$\mathrm{d} u=u_{0} \exp (\lambda t) J_{m}(\kappa r) \exp (\mathrm{i} m \theta)$,

$\mathrm{d} v=v_{0} \exp (\lambda t) J_{m}(\kappa r) \exp (\mathrm{i} m \theta)$.

By substituting these solutions in (2), we obtain

$$
\begin{aligned}
& \lambda u_{0} J_{m}(\kappa r) \exp (\mathrm{i} m \theta) \\
& \quad=\left[D \delta u_{0} \nabla^{2}+\left(\alpha u_{0}+v_{0}\right)\right] J_{m}(\kappa r) \exp (\mathrm{i} m \theta), \\
& \lambda v_{0} J_{m}(\kappa r) \exp (\mathrm{i} m \theta) \\
& \quad=\left[\delta v_{0} \nabla^{2}+\left(\beta v_{0}-\alpha u_{0}\right)\right] J_{m}(k r) \exp (\mathrm{i} m \theta) .
\end{aligned}
$$

For simplicity, let us consider symmetrical modes only, then by taking into account that in polar coordinates, $\nabla^{2}\left(J_{m}(\kappa r) \exp (\mathrm{i} m \theta)\right)=-\kappa^{2} \exp (\operatorname{i} m \theta) J_{m}(\kappa r)$, we obtain

$\left(\lambda+D \delta \kappa^{2}-\alpha\right) u_{0}-v_{0}=0$,

$\alpha u_{0}+\left(\lambda+\delta \kappa^{2}-\beta\right) v_{0}=0$.

Nontrivial solutions are obtained if

$\left(\lambda+D \delta \kappa^{2}-\alpha\right)\left(\lambda+\delta \kappa^{2}-\beta\right)+\alpha=0$.

Therefore

$$
\begin{aligned}
& \lambda^{2}+\left[(1+D) \delta \kappa^{2}-\beta-\alpha\right] \lambda \\
& \quad+\left[\left(D \delta \kappa^{2}-D \beta-\alpha\right) \delta \kappa^{2}+\alpha(\beta+1)\right]=0 .
\end{aligned}
$$

Observe that this dispersion relation is exactly the same as the one for a square domain [10], except that the plane wavenumber $|\mathbf{k}|=k$ plays the role of $\kappa$ [7]. Therefore, for zero-flux boundary conditions

$k=\frac{\kappa_{m n}}{a}$,

where $\kappa_{m n}$ is the $n$th zero of the derivative of the Bessel function $J_{m}^{\prime}$ and $a$ the radius of the disk.
The bounds in $\mathbf{k}$ space of a region with positive $\lambda$ occur when $\lambda\left(k_{\mathrm{c}}^{2}\right)=0$. Then, the independent term of (4) must be zero and $k_{\mathrm{c}}$ satisfies

$$
\left(D \delta k^{2}-D \beta-\alpha\right) \delta k^{2}+\alpha(\beta+1)=0,
$$

or

$$
D \delta^{2} k^{4}-(D \beta+\alpha) \delta k^{2}+\alpha(\beta+1)=0 .
$$

If we wish to select a single excited wavevector, we require the previous equation to have only one solution for $k_{\mathrm{c}}^{2}$, which occurs when

$$
(D \beta \delta+\alpha \delta)^{2}-4 D \delta^{2} \alpha(\beta+1)=0 .
$$

Then, the modulus of the critical wavevector is

$$
\begin{aligned}
k_{\mathrm{c}}^{2} & =\frac{1}{2 D \delta^{2}}(D \beta \delta+\alpha \delta) \\
& =\frac{1}{2 D \delta^{2}} \sqrt{4 D \delta^{2} \alpha(\beta+1)}=\frac{1}{\delta} \sqrt{\frac{\alpha(\beta+1)}{D}} .
\end{aligned}
$$

Observe that this equation implies that once $(a, \alpha$, $\beta, D)$ are fixed, one could select a pattern of a given radial symmetry by varying $\delta$. Alternatively, solving (6) for $\delta$, one could tune the other parameters to satisfy condition (5) for a given symmetry $m$. Other boundary conditions might be considered on the same footing. For instance, for fixed boundary conditions, Eq. (5) still holds, if $\kappa_{m n}$ is the $n$th zero of $J_{m}$. One might consider more complicated problems, such as having an inhomogeneous domain, for instance, a disk where $D=D_{\mathrm{i}}$ embedded in a square where $D=D_{\mathrm{o}}$. Such a system has been studied in one dimension by one of us [11], and it has been shown that the dispersion relation does not admit an analytic solution, even in the simple case of a step-like variation of the diffusion constant and zero-flux external conditions. In general, the Laplacian should be replaced by $\nabla \cdot(D(x, y) \nabla u)$. It was also shown that for certain values of the bifurcation parameter different solutions are confined in each part of the inhomogeneous system. In two dimensions that would imply that we can use our simple solution (6) for the disk, and apply it equally to the wavevector of the solution for the outer square domain, taking care of matching both lengths at the border between 
regions. That would mean that the condition to produce a mode of symmetry $m$ inside the disk is

$\left(\frac{k_{0}}{\kappa_{m n}}\right)^{2}=\frac{1}{a} \sqrt{\frac{D_{\mathrm{i}}}{D_{\mathrm{o}}}}$.

As we shall see in the next section, Eqs. (6) and (7) predict the symmetry of the patterns obtained in numerical calculations.

\section{Numerical calculations}

The first method used to solve Eqs. (1) is similar to the one explained in Ref. [12]. Basically one discretizes the Laplacian in polar coordinates, the central points are joined in pairs separated by $\theta=\pi / 2$, and at the outer points in the circumference, fixed boundary conditions are imposed. In Fig. 1, a calculation in a lattice of 68 points in $\theta$ and 34 points in $r$ is shown. The parameters used were chosen as in Ref. [10] in order to obtain a robust pattern of spots, namely $\alpha=$ $0.899, \beta=0.91$, and $D=0.516$, with $r_{1}=r_{2}=0.2$. The value of $\delta=0.005$ was obtained from Eq. (6) by choosing the first zero of $J_{5}$ and by considering a unitary disk. We used 6000000 time iterations to converge, with a time step of $\mathrm{d} t=0.0001$. A small variation of $\delta=0.004$ produces the hexagonal pattern also shown in the figure.

When one imposes zero-flux boundary conditions on the rim, one obtains similar patterns, although the values of $\delta$ needed to produce a regular symmetry vary slightly, since one has to seek for the zeros of $J_{m}^{\prime}$. Examples of patterns obtained with zero-flux boundary conditions are shown in Fig. 2. The important point to remark here is that one is able to obtain perfectly regular patterns only by varying the size of the domain. It seems that the crucial feature is the geometry of the boundary. This effect of the boundary is expected to be lost when the size of the domain is large as compared to the typical wavelength of the pattern. The centered symmetry is due to the selection of only a few admissible eigenmodes.

Notice the appearance of the central spot in some of the patterns in Fig. 2. In particular, consider the pentagonal pattern. Since $J_{5}\left(k_{51} r\right)$ is zero at the origin, we should expect zero values there, which is not the case. We should, however, note that the linear analysis predicts excited modes under the linear regime, while the final solution depends also on the nonlinear terms and it is expected that, besides mono-modes, nonlinear
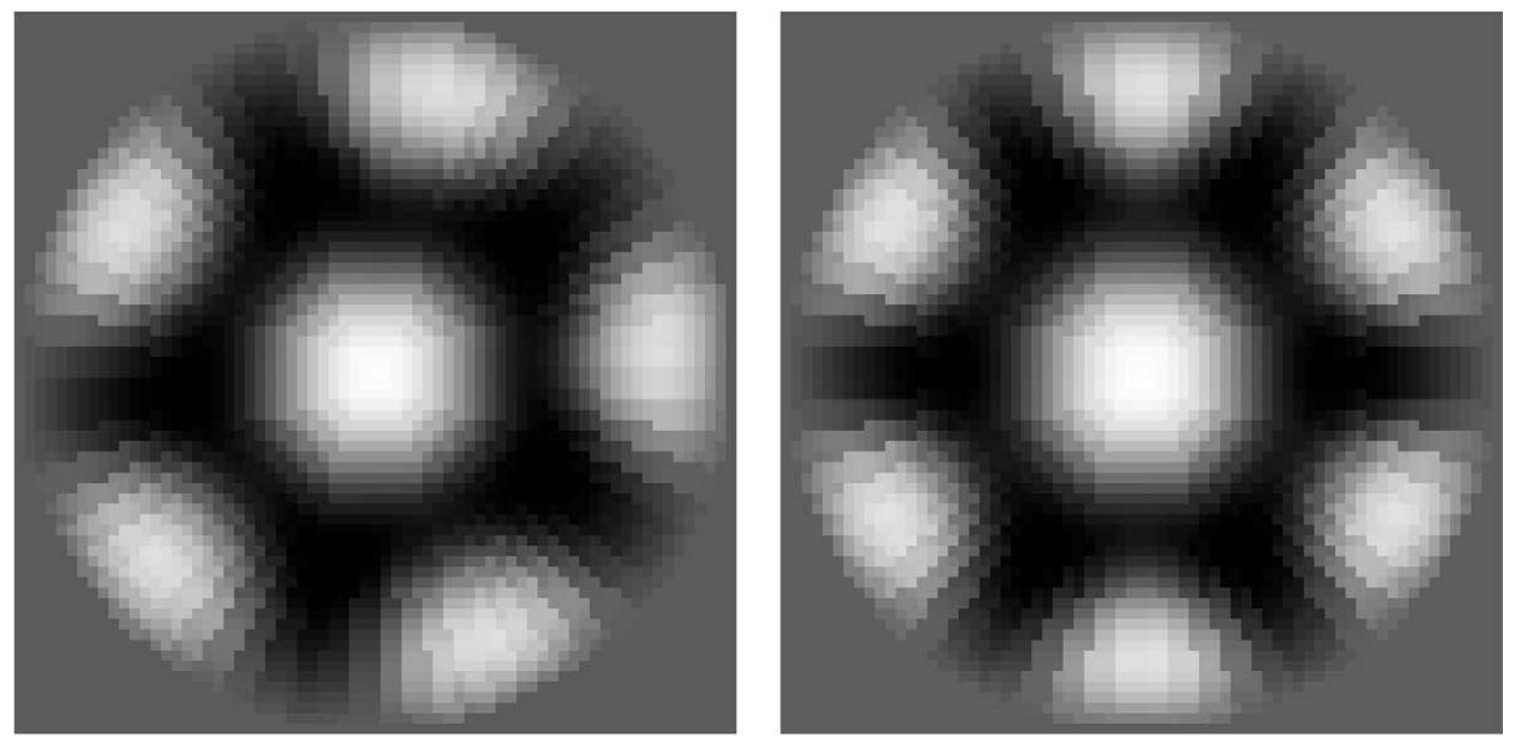

Fig. 1. Fivefold and sixfold regular patterns obtained in a disk using polar coordinates with fixed boundary conditions. 

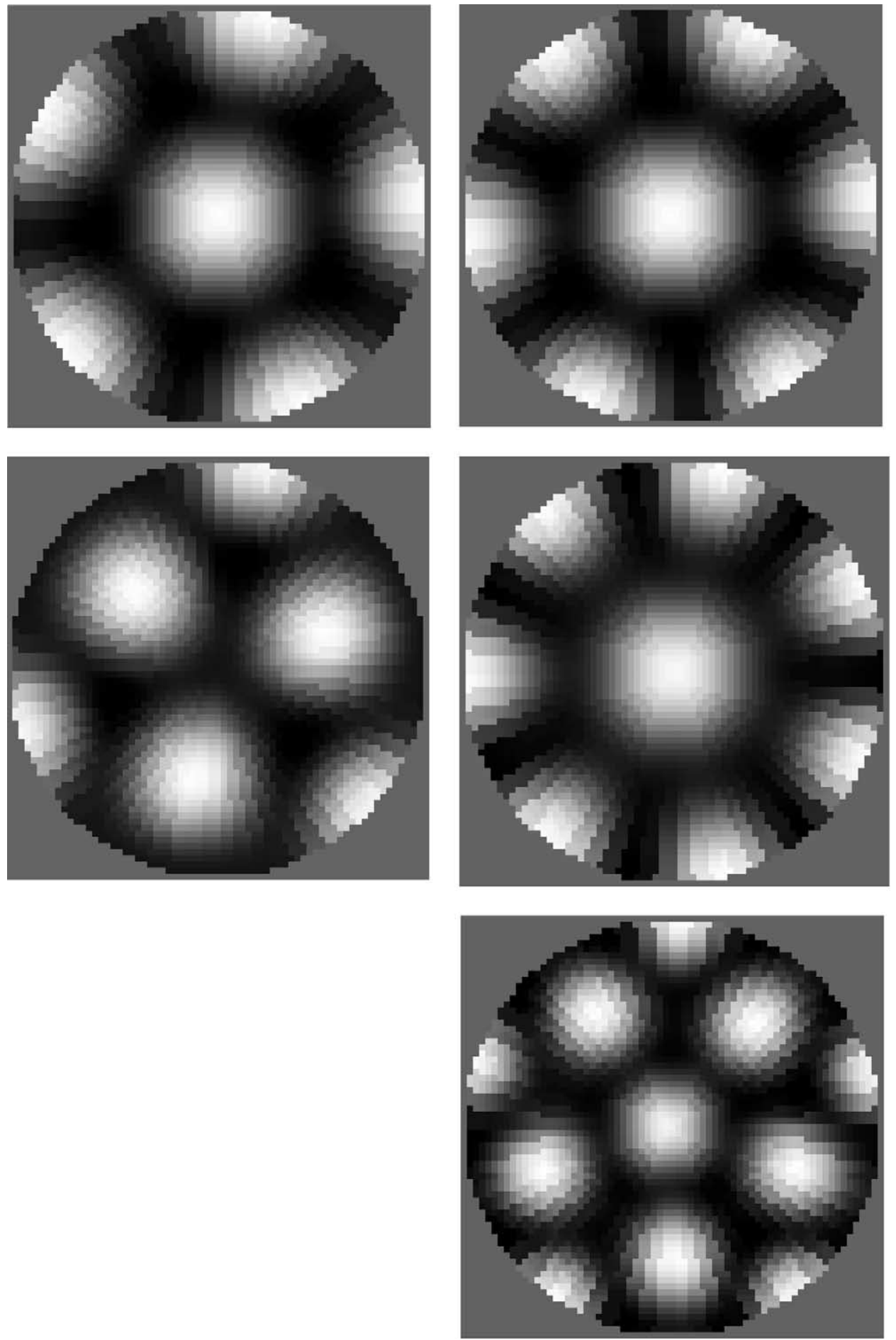

Fig. 2. Series of symmetries obtained in a disk with zero-flux boundary conditions. The values of $\delta$ are: $0.009,0.0083,0.0064,0.0062$, 0.0034. These correspond to increasing the size of the domain. 


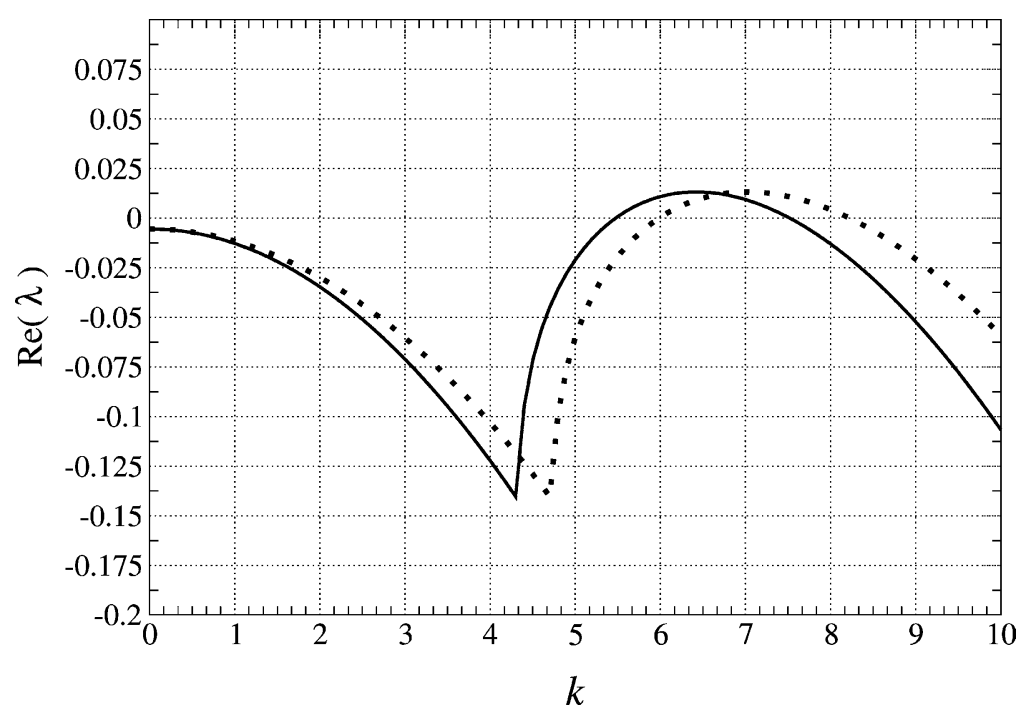

Fig. 3. Variation of dispersion relation (4) for two different parameter values. Continuous line corresponds to $\alpha=0.899, \beta=-0.91$, $D=0.516$, and $\delta=0.00962$, to enhance the mode $k_{51}=6.41562$. Dotted line corresponds to the same parameters as in the previous case but $\delta=0.00804$ to enhance mode $k_{03}=7.01558$.

coupling of different modes may appear. In Fig. 3, the dispersion relation (4) is plotted for two different parameter values, in the appropriate parameter domain. The parameters were chosen to enhance modes $k_{51}=$ $\kappa_{51}=6.41562$ and $k_{03}=\kappa_{03}=7.01558$, according to (6). From the figure, we observe that at the range of $k$ where the mode 51 is enhanced, also the mode 03 is excited and so it may also be present. This is in fact what occurs; a modal decomposition of the pentagonal pattern shown in Fig. 2 reveals that modes 03 and 01 are blending into 51 [13].

In order to verify that the regularity of the patterns (they almost always present radial symmetry) is not produced by a numerical artifact when using polar coordinates, we solved the equations in a square domain using Cartesian coordinates, exactly as done in Ref. [10]. There is a great deal of literature on bifurcation analysis and numerical simulations of this problem on square domains showing how different types of hexagons arise [14].

We choose a grid of $32 \times 32$ and defined a centered disk with diameter 28 and $\delta=2$. We used $r_{1}=0.5$ and $r_{2}=0.4$. Inside the disk we set $D_{\mathrm{i}}=0.51$, for the remaining part of the square domain $D_{\mathrm{o}}=0.516$, and we suitably modify the diffusion terms of the model equations to enforce conservation of flux. In Fig. 4, we show a nearly perfect pentagonal pattern, after 400000 time steps of 0.05 , starting with random initial conditions. If one removes the circular boundary by making $D_{\mathrm{i}}=D_{\mathrm{o}}=0.516$, one obtains an hexagonal pattern that is not centered. Again this result supports the idea that regular patterns appear when a circular inhomogeneity is present. Only a small change in the homogeneity of the square domain seems to be enough to produce a centered symmetrical pattern. Of course, we expect that when the size of the disk is large enough one recovers the usual hexagonal pattern.

Former studies in one dimension of a Schakenberg system with a step-like variation of the diffusion constants [11] show that there are three types of patterns, depending on the value of the ratio $D_{\mathrm{o}} / D_{\mathrm{i}}$. Roughly speaking, when this ratio is large, isolated patterns (type A) are obtained in each region, and there is a discontinuity at the border. When this ratio diminishes, there are isolated patterns, but the transition at the border of the domains is smooth (type B patterns), and finally, when the ratio approaches 1 , the type B patterns become non-isolated. We expect that a similar situation still holds in this model and in two dimensions. The patterns shown in Fig. 4 are surely non-isolated 

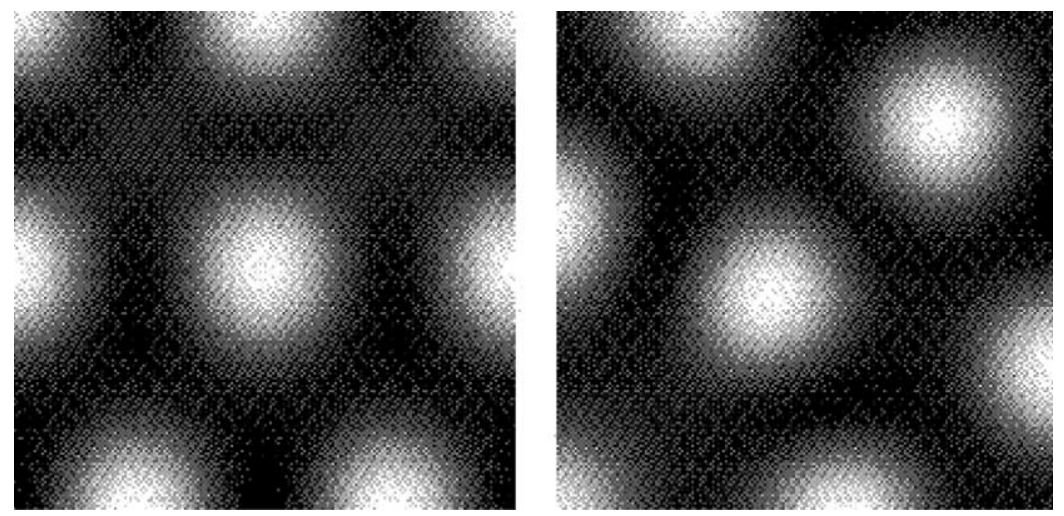

Fig. 4. Fivefold symmetry pattern in a square domain with $D_{\mathrm{o}}=0.516$, obtained by defining a disk inscribed in the square, in which $D_{\mathrm{i}}=0.510$. If the square domain is homogeneous $\left(D_{\mathrm{i}}=D_{\mathrm{o}}\right)$, the usual hexagonal array is obtained.

and we have to test the results in an isolated type regime. In order to do this, we solve the equations in an inhomogeneous domain consisting of a disk embedded at the center of a square domain, using a grid that allows for a variation of the size of the disk, and set $D_{\mathrm{o}}=0.3$ and $D_{\mathrm{i}}=0.516$. In Fig. 5, we show the results of a calculation on a grid of $42 \times 42$ with $\delta=2$. Obviously, if the size of the disk is small enough to prevent the appearance of $J_{0}$, no pattern is observed inside the disk, but the number of spots surrounding the disks is regular, and the spots have to deform themselves in order to match this regularity.

We continue growing the disk by using a larger grid of $68 \times 68$. In Fig. 6 , we show a sequence of patterns, starting with a disk large enough for a central spot to fit in. Observe that when a further layer of spots forms, the symmetry of the pattern is 5 . The hexagonal pattern is more robust. In some calculations the initial symmetry breaking could be 5 or 7 , but as the pattern converges and becomes stable, a sixfold pattern finally settles in.

We want to investigate the sequence of patterns for larger disks, but numerical calculations on larger grids become exceedingly lengthy. We take advantage of the fact that $\delta$ is a parameter that only sets the scale of the wavevector as compared to the grid division. In Fig. 7, we show a series of symmetrical patterns on a square grid of $68 \times 68$ and $\delta=0.48$. This value means that the linear dimension of the domain is approximately doubled. We show the appearance of a third and a fourth layer of spots inside the disk. Observe that the sequence is 3-4-5-6-7, and that when the central spot disappears, a new series starts, and when it reappears, the pattern is fivefold.

It is possible to imagine other types of inhomogeneity within this model. For instance, one could define a
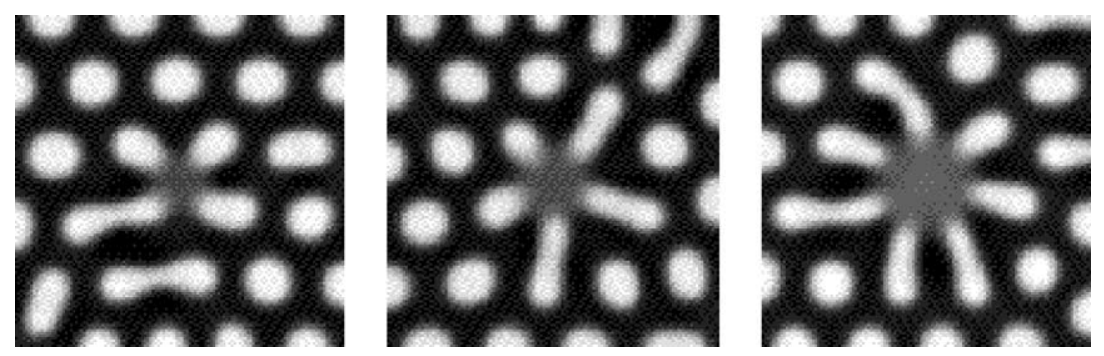

Fig. 5. Patterns obtained for a very small disk with different $D$ in the center of the square; see the text for information about the parameters. The disk grows from left to right. 

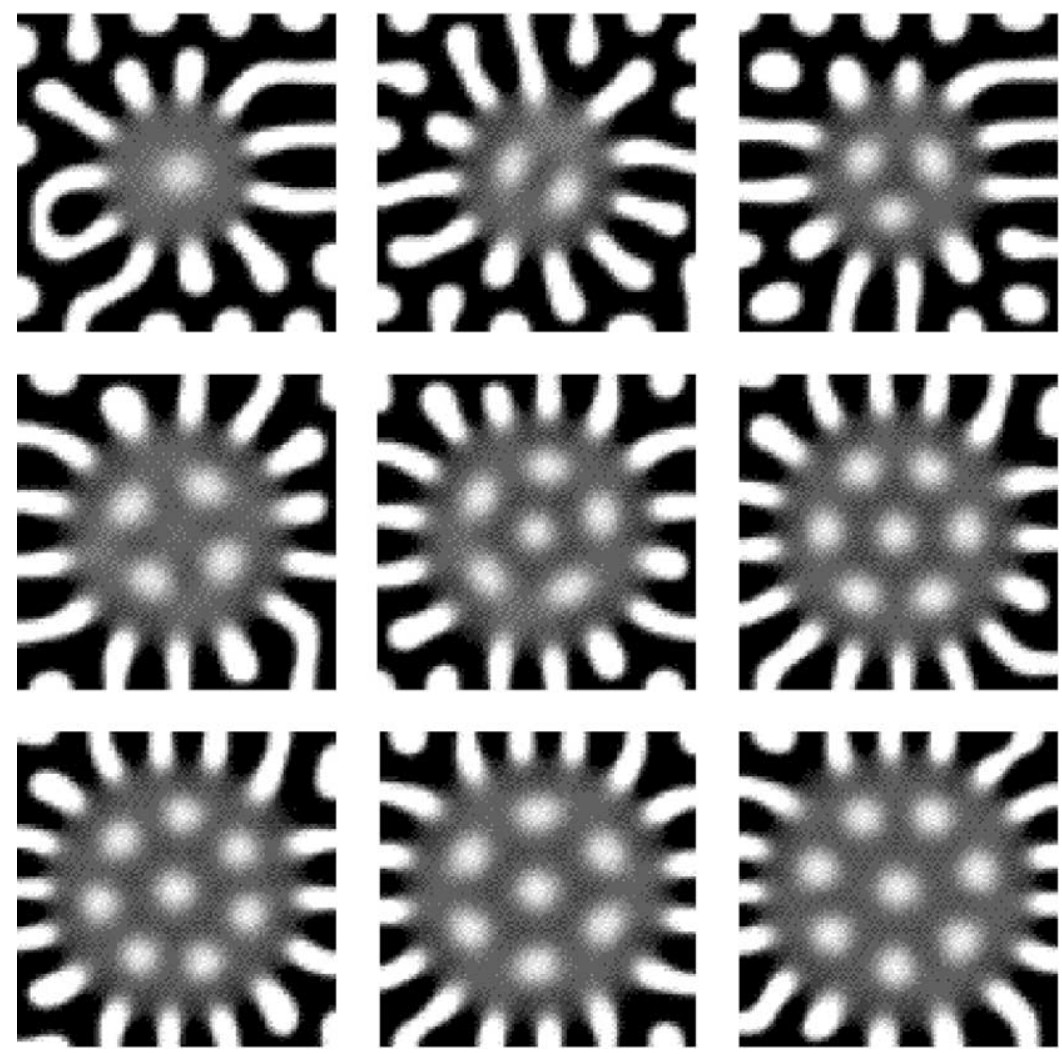

Fig. 6. As the previous figure, except that the central disk has reached the minimum size to allow for the first zero of $J_{0}$. Notice that when a new layer of spots appears the symmetry is fivefold. Also notice the re-entrant symmetry 6 in the bottom row.
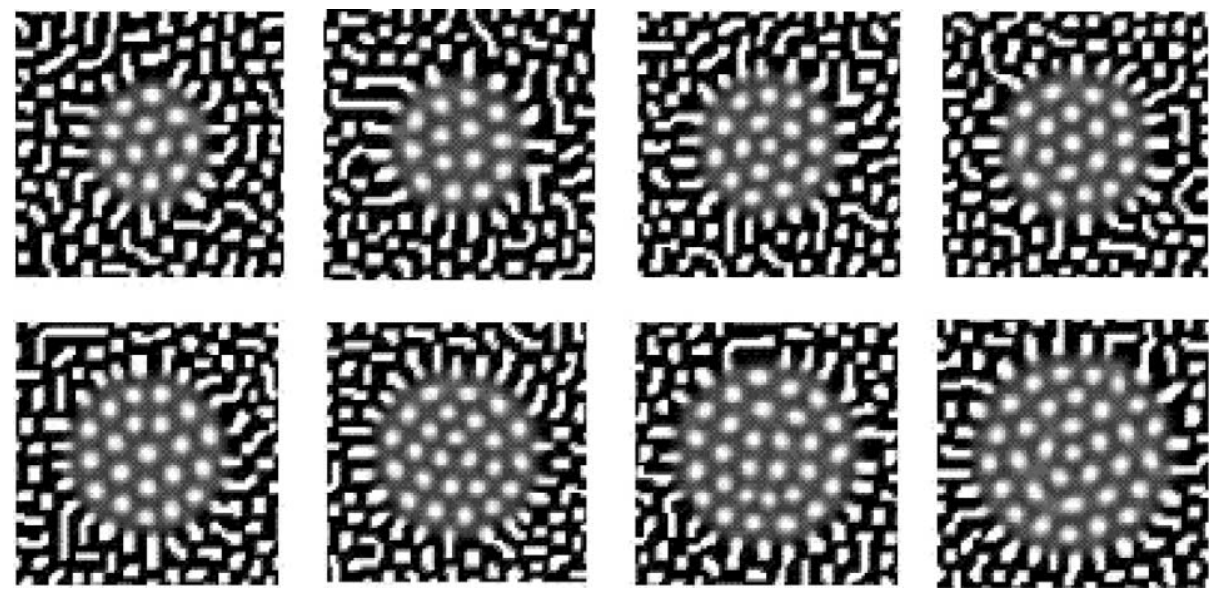

Fig. 7. As the size of the disk increases, the series of symmetries $(3 \rightarrow 7)$ repeats itself. For larger sizes, the effect of the boundary is lost and one recovers the usual hexagonal array of spots. 
(a)

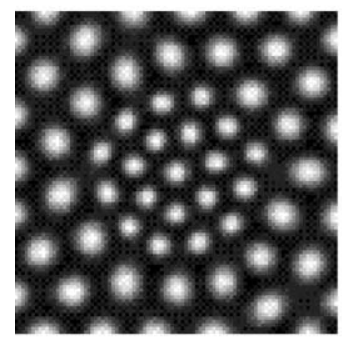

(c)

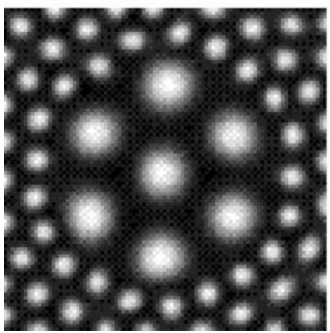

(b)

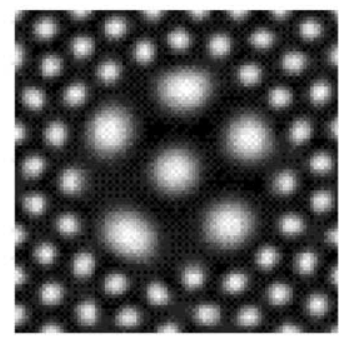

(d)

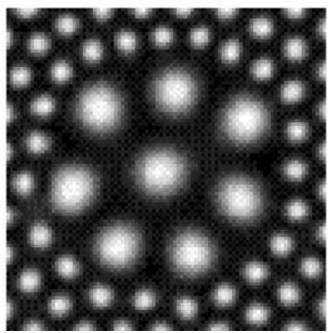

Fig. 8. (a) Sevenfold pattern obtained in a $64 \times 64$ grid with $\delta_{\text {out }}=1$. Inside the disk $\delta_{\text {in }}=0.5$. Patterns in (b)-(d) show a series of symmetries obtained by increasing the size of the disk, and maintaining $\delta_{\text {in }}=0.5$ and $\delta_{\text {out }}=2$.

different $\delta$ inside the circular domain, and expect that the size of the spots is modified. Fig. 8 illustrates the results of a numerical calculation varying the value of $\delta$. The remarkable thing is that regular patterns are also obtained and that they follow the same size-dependent symmetry.

These patterns resemble the situation found in the embryogenesis of the skin, where the precursors of scales, feathers, or hair, form a complicated pattern of large and small spots. Complex patterns like these have been obtained before by coupling a reaction-diffusion model to a mechano-chemical model [15]. It is difficult to find a biological basis to assume that a pre-pattern of different diffusion properties is formed in a real tissue, but no more unlikely than the precise temporal changes dictated by genetics, often proposed to model the superposition of two wave-like patterns.

We have also investigated inhomogeneities in other parameters; for instance, a variation of $\beta$ also produces regular patterns. We observed that the robustness of spots is overwhelming, even when the nonlinear parameters were set to produce stripes $\left(r_{2}=0\right)$, inside the disk the symmetrical array of spots was always present. This might explain why in our model for the Faraday experiment [4] we obtain asymmetric spots patterns, even though the lowest nonlinearity in the model is cubic, favoring the formation of stripes. Preliminary calculations using a model that couples a chemotactic mechanism to a Turing model [16] show a similar series of symmetric patterns, although not as regular as the ones presented here. Given the mathematical similarities of a wide class of models proposed for morphogenesis, we expect our results to hold more generally.

\section{Discussion}

The pattern with spots exhibiting hexagonal symmetry is ubiquitous in Turing models for biological morphogenesis [14,17-19]. A weakly nonlinear theory based on corresponding amplitude equations has been recently developed to study the stability of this unavoidable hexagonal pattern [20]. Unfortunately, for Turing pattern selection, a nonlinear theory foreseeing different symmetries similar to that found for Faraday wave patterns [21] does not exist up to date. In the strategy of the present paper, we apply simple linear theory and the geometric frustration concept to reduce the usual hexagonal symmetry of the Turing patterns and to produce the biologically important fivefold patterns in small-size disks. When the disk size increases or the pattern grows in wider domains out of the disk, the above-mentioned nonlinear mechanism of Turing pattern selection [20] recovers its major role and the hexagonal symmetry ubiquitously returns. Starting from the idea of linear patterns confined in small disks, one can imagine non-hexagonal seeds of biological forms that could be frozen at an early stage of development in embryos. This idea has been recently applied by us to study the de novo appearance of the five primary podia during the development of regular echinoid larval forms [13]. During the metamorphosis of larvae, these animals develop five buds of primary podia in a disk called the imaginal rudiment or rudiment disk [22]. These five podial buds play the main role during the embryological skeletal development of echinoids 

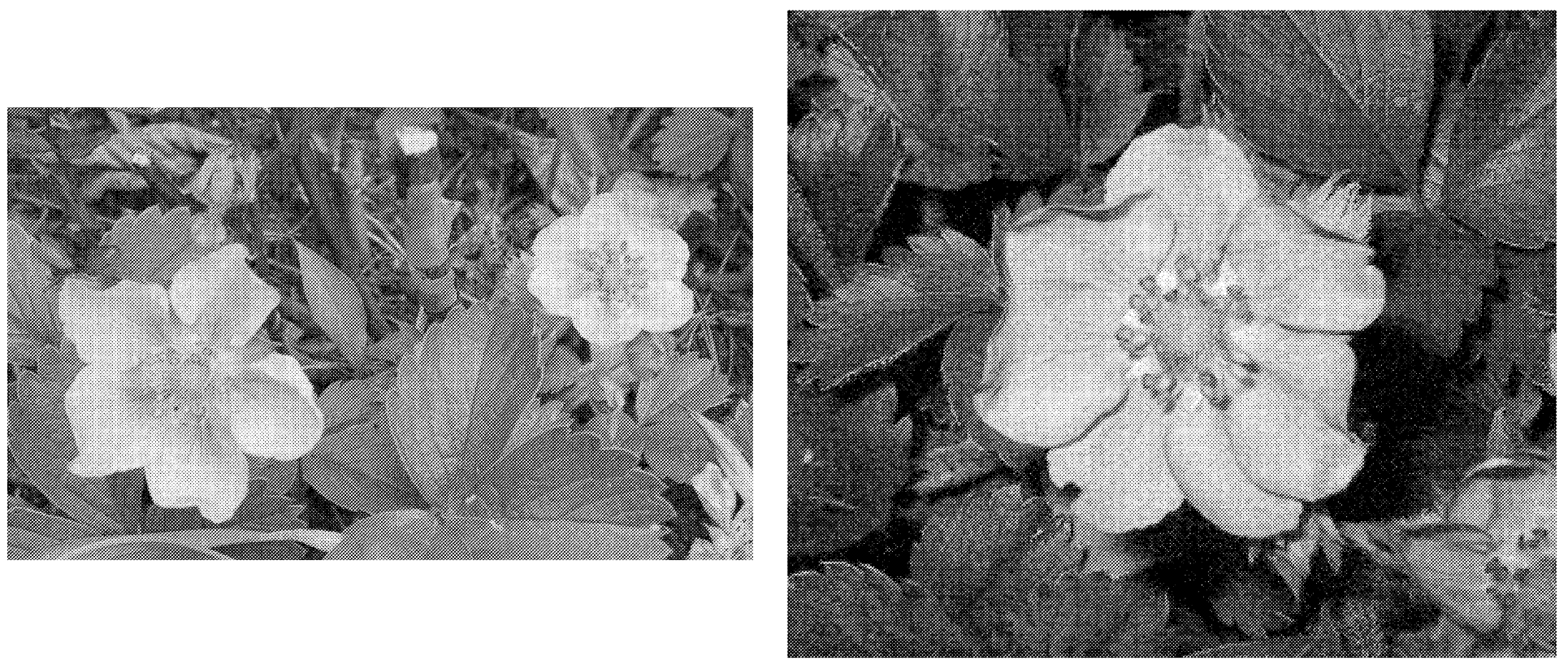

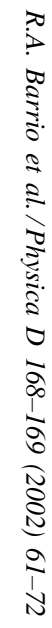

Fig. 9. Fivefold, sixfold, and sevenfold flowers found in the same strawberry plant. 
[23]. Notice that, from a genetic point of view, the pentagonal symmetry of the adult echinoid can be efficiently and robustly coded by using only two basic parameters as highly condensed instructions: the radius of the rudiment disk (i.e., the cell division number) and the characteristic wavelength corresponding to the morphogens. This could explain why the symmetry is robustly kept when it is generated starting from a disk-like embryological structure, as in the case of the sea urchins. On the contrary, when the primordia grow starting from a symmetry breaking along a string-like one-dimensional structure, as in the case of leaf and flower initiation during plant growth [17], the final symmetry could not be controlled in an unique way. For instance, a single strawberry plant can produce flowers with a variable number of petals (see below).

\section{Conclusions}

In this work, we have studied numerically a simple Turing model in small circular domains with various boundary conditions. The results show that centered symmetric patterns of spots are always robust, and that the symmetry of the pattern depends on the size of the circular domain. Linear analysis of the simplest cases shows that this result is due to a precise selection of eigenmodes during the onset of the instability when the size of the disk is comparable to the distance at which the lower-order Bessel functions (or their derivatives) have their first zeros. As the size of the circular domain becomes large, the higher-order zeros of the modes become closer together and many more modes become excited under diffusion, the patterns lose radial symmetry and the effect or the border is negligible. This phenomenon was verified in various inhomogeneous domains, and the series of size-dependent symmetric patterns appears to be very similar in all cases, as far as a small circular border exists.

This main result is extremely important for models of morphogenesis, since it shows that simple Turing mechanisms are capable of producing complex patterns of a given symmetry, usually found in real organisms. Nature strikes us not only by its beautiful diversity of patterns, but by the seemingly universal way in which few simple patterns appear. In some cases the symmetry is robustly kept, as in the case of the morphogenesis of echinoderms. In other cases the symmetry is not tightly controlled; for instance, a single strawberry plant can produce flowers with five, six, or seven petals (see Fig. 9). Other interesting examples of this can be found in Ref. [7].

This implies that the problem originally posed by Turing, of forming an anatomical structure from a zygote, is not solved only by forming a pattern with spatial information, but additionally, there should be a mechanism capable of controlling the bifurcation path of the model. As the study of genetics is not yet providing us with such a mechanism, this paper shows that domain size and shape could provide it.

Two possible factors that might affect pattern selection are the growth and the curvature of the domain, because real organisms really generate from growing three-dimensional curved objects. A study of the effect of these factors in the selection of Turing patterns is currently being carried out [24].

\section{Acknowledgements}

This work was supported by DGAPA UNAM through projects IN104598 and IN108199. RAB wants to thank the Department of Theoretical Physics, Oxford University, for their hospitality during the preparation of this work.

\section{References}

[1] M.I. Rabinovich, A.B. Ezersky, P.D. Weidman, The Dynamics of Patterns, World Scientific, Singapore, 2000 (Chapter 1).

[2] M. Faraday, Phil. Trans. R. Soc. London 121 (1831) 299.

[3] M.I. Rabinovich, A.B. Ezersky, P.D. Weidman, Phil. Trans. R. Soc. London 121 (1831) 25.

[4] R.A. Barrio, J.L. Aragón, C. Varea, M. Torres, I. Jiménez, F. Montero de Espinosa, Phys. Rev. E 56 (1997) 4222.

[5] M. Torres, G. Pastor, I. Jiménez, F. Montero de Espinosa, Chaos, Solitons and Fractals 5 (1995) 2089.

[6] A.M. Turing, Phil. Trans. R. Soc. London B 237 (1952) 3772.

[7] J.D. Murray, Mathematical Biology, second ed., Springer, Berlin, 1993. 
[8] P.K. Maini, K.J. Painter, H.N.P. Chau, J. Chem. Soc., Faraday Trans. 93 (1997) 3601.

[9] T. Leppänen, M. Karttunen, K. Kaski, R.A. Barrio, L. Zhang, Physica D 168-169 (2002) 35-44.

[10] R.A. Barrio, C. Varea, J.L. Aragón, P.K. Maini, Bull. Math. Biol. 61 (1999) 483.

[11] D.L. Benson, J.A. Sherratt, P.K. Maini, Bull. Math. Biol. 55 (2) (1993) 365.

[12] C. Varea, J.L. Aragón, R.A. Barrio, Phys. Rev. E 60 (4) (1999) 4588

[13] J.L. Aragón, M. Torres, D. Gil, R.A. Barrio, P.K. Maini, Phys. Rev. E, 65 (2002) 051913.

[14] D.J. Wollkind, L.E. Stephenson, in: P.K. Maini, H.G. Othmer (Eds.), Mathematical Models for Biological Pattern Formation, Springer, Berlin, 2000, pp. 113-142 and references therein.

[15] L.J. Shaw, J.D. Murray, SIAM J. Appl. Math. 50 (2) (1990) 628.
[16] K.J. Painter, P.K. Maini, H.G. Othmer, Proc. Natl. Acad. Sci. USA 96 (1999) 5549.

[17] A.J. Koch, H. Meinhardt, Rev. Mod. Phys. 66 (1994) 1481.

[18] J. Boissonade, E. Dulos, P. De Kepper, in: R. Kapral, K. Showalter (Eds.), Chemical Waves and Patterns, Kluwer Academic Publishers, Dordrecht, 1995, p. 221.

[19] P. Borckmans, G. Dewel, A. Dewit, D. Walgraef, in: R. Kapral, K. Showalter (Eds.), Chemical Waves and Patterns, Kluwer Academic Publishers, Dordrecht, 1995, p. 323.

[20] B. Peña, C. Pérez-García, Europhys. Lett. 51 (2000) 300.

[21] P. Chen, J. Viñals, Phys. Rev. Lett. 79 (1997) 2670.

[22] R.B. Emlet, Biol. Bull. 174 (1988) 4; A.V. Spirov, J. Theoret. Biol. 161 (1993) 491.

[23] B. David, R. Mooi, in: R. Mooi, M. Telford (Eds.), Echinoderms: San Francisco, Balkema, Rotterdam, 1998, p. 21.

[24] P. Padilla, R. Plaza, F. Sánchez-Garduño, R.A. Barrio, P.K. Maini, in preparation. 